\title{
Performance Comparison of Various Filters and Wavelet Transform for Image De-Noising
}

\author{
Chanchal Srivastava ${ }^{1}$, Saurabh Kumar Mishra ${ }^{2}$, Pallavi Asthana ${ }^{3}$, G. R. \\ Mishra $^{4}$, O.P. Singh ${ }^{5}$ \\ Department of Electronics \& Communication Engineering Amity School of Engineering \& Technology \\ Amity University Uttar Pradesh Lucknow Campus, India
}

\begin{abstract}
Digital images are prone to a variety of noise. The varieties include Speckle noise, Gaussian noise, Salt and pepper noise. It is a difficult task to separate noise from an image while maintaining the desired information and quality of an image. To obtain significant results, various algorithms have been proposed. This paper deals with comparison of two approaches i.e. filtering approach and wavelet based approach accounting Peak Signal to Noise Ratio and Root Mean Square Error as performance parameters. This paper proves that Wavelet Transform method is very effective for all types of noise. Results of this paper have been simulated on MATLAB.

Keywords - Median filter, Average filter, Wiener filter, Gaussian filter, Speckle noise, Gaussian noise, Salt \& Pepper noise Wavelet Transform.
\end{abstract}

\section{INTRODUCTION}

The area of digital image processing belongs to processing of digital images by using digital computer [1]. Digital images are form of visual information captured or transmitted using camera or other imaging system. The received image might be corrupted due to the presence of noise. It becomes necessary to bring out the original image before applying to different applications [5]. Various kinds of noises exist in an image and a variety of noise reduction techniques are available to perform de-noising. Selection of the de-noising algorithm depends on the application. Gaussian noise, speckle noise, salt \& pepper noise, shot noise are types of noises that are present in an image. The principle approach of image de-noising is filtering. Available filters to de-noise an image are median filter, Gaussian filter, average filter, wiener filter and many more. The resulting image from this approach is either blurred or over smoothed due to losses. To overcome the drawback of filtering approach, a mathematical function is developed known as wavelet transform. It decomposes an image into its frequency components and subsequently reconstructs with high precision [3]. The results from this method are more effective than former.

\section{MEDianFilter}

The median filter is a non-linear digital filtering technique. It proves to be best in removing salt and pepper noise and impulse noise. Median filter erases black dots called the pepper and fills in white holes in the image, called salt. It better works than mean filter by preserving sharp edges. It simply replaces each pixel value by the median of the intensity level in the neighborhood of that pixel [1].

\section{WIENER FILTER}

Wiener filters are a class of optimum linear filters. It provides linear estimation of a desired signal sequence from another related sequence[10]. The wiener filter provides a solution of signal estimation problem for stationary signals. It also provides successful results in removing noise from photographic image. The design of the filter is distinct. It is based on statistical approach. The filter is optimal in the sense of MMSE.

\section{GAUSSIAN FILTER}

Gaussian filters are a class of linear smoothing filters. The weights are chosen according to the shape of Gaussian function. The Gaussian smoothing filter is a very good filter to remove noise drawn from a normal distribution. The Gaussian filter is non-causal i.e. the filter window is symmetric about the origin in time domain. Because of this property Gaussian filters are physically unrealizable.

\section{AVERAGe Filter}

Average filter or mean filter is simple, instinctive and easy to understand. It performs smoothing of images i.e. reducing the amount of intensity variation between one pixel and the next. Each pixel value in an 
image is replaced with the average value of its neighbors, including that pixel. This has the effect of eliminating pixel values which are not representative of their surroundings.

\section{IMAGE NOISE}

Noise in an image is the result of errors in the image acquisition process that result in pixel values that do not reflect the true intensities of real picture. The presence of noise gives an image a grainy, rough, mottled or snowy appearance. The magnitude of image noise can vary from almost gradual specks on a digital photograph to opticalandradioastronomicalimages that are completely noise. Various types of noises present in an image are as follows:

* Gaussian noise

* Salt and pepper noise

* Speckle noise

\section{A. Gaussian Noise}

Gaussian noise is a statistical noise. It is evenly distributed over the signal [5]. It is a major part of 'read noise' of an image sensor i.e. of the constant noise level in dark areas of the image [4][10]. The probability density function (PDF) of Gaussian noise is equal to that of the normal distribution, also known as Gaussian distribution. It is usually used as additive white noise to give additive white Gaussian noise (AWGN).

\section{B. Salt and Pepper Noise}

Fat-tail distributed or impulsive noise is sometimes called salt and pepper noise or spike noise. An image containing salt and pepper noise will have dark pixels (black dots or pepper) in bright region and bright pixels (white dots or salt) in dark region [4][10]. An effective method to remove this type of noise involves the use of median filter, morphological filter or a contra harmonic median filter.

\section{Speckle Noise}

Speckle noise is a granular noise that inherently exists in and degrades the quality of the active radar and synthetic aperture radar (SAR) images. Speckle is a random, deterministic, interference pattern in an image formed with coherent radiation of a medium containing many sub-resolution scatterers. Speckle noise is eliminated using adaptive and non-adaptive filters.

\section{WAVELET TRANSFORM}

Wavelet analysis is a new development in the area of applied mathematics. Wavelets are mathematical functions that allow complex information to be decomposed into different frequency components, and then study each component with a resolution matched to its scale. It is a complement to the classical Fourier transform. Fourier analysis is ideal for studying stationary data but it does not work well for studying data with transient events. Wavelet transforms are particularly useful for aperiodic, noisy, non-continuous, and transient type of signals [2]. They have special ability to examine signals simultaneously in both time and frequency. Wavelet means small waves [3]. The wavelets can be built by taking adifferent shape, called a mother wavelet, and dilating, compressing or shifting it in time. They are classified as continuous wavelet transforms (CWTs) and discrete wavelet transforms (DWTs). Wavelets with their generality and strong results have quickly become useful to a number of disciplines. Current application of wavelet includes climate analysis, heartmonitoring, seismic signal de-noising, compression, crack surface characterization and so on [6].

\section{PARAMETRIC DESCRIPTION}

The performance parameters are most important criteria to justify results through evaluation. The parameters considered here are peak signal to noise ratio (PSNR) and mean square error (MSE). The objective quality of the reconstructed image is measured by:

$$
P S N R=10 \log _{10}\left(\frac{R^{2}}{M S E}\right)
$$

Where $\mathrm{R}$ is maximum value of the pixel present in an image and MSE is mean square error between the original and de-noised image with size $M * N[9]$. Mean square error is defined as:

$$
M S E=\frac{1}{M * N} \sum_{i=1}^{M} \sum_{j=1}^{N}[x(i, j)-y(i, j)]^{2}
$$

Where, $x(i, j)$ is original image and $y(i, j)$ is de-noised image. Root mean square error is defined as:

$$
R M S E=\operatorname{sqrt}(M S E)
$$

i.e. root mean square error is square root value of mean square error. 


\section{SimUlation ReSUlts}

A gray scale image (Lena) is taken as reference image. Three types of noise i.e. Gaussian noise, salt and pepper noise and speckle noise are added to the image. De-noising is performed using four filters i.e. Median filter, Wiener filter, Gaussian filter, Average filter and with Wavelet Transform. Results are shown through comparison among them. Comparison is being made on the basis of some evaluated parameters. The parameters are peak signal to noise ratio (PSNR) and root mean square error (RMSE).

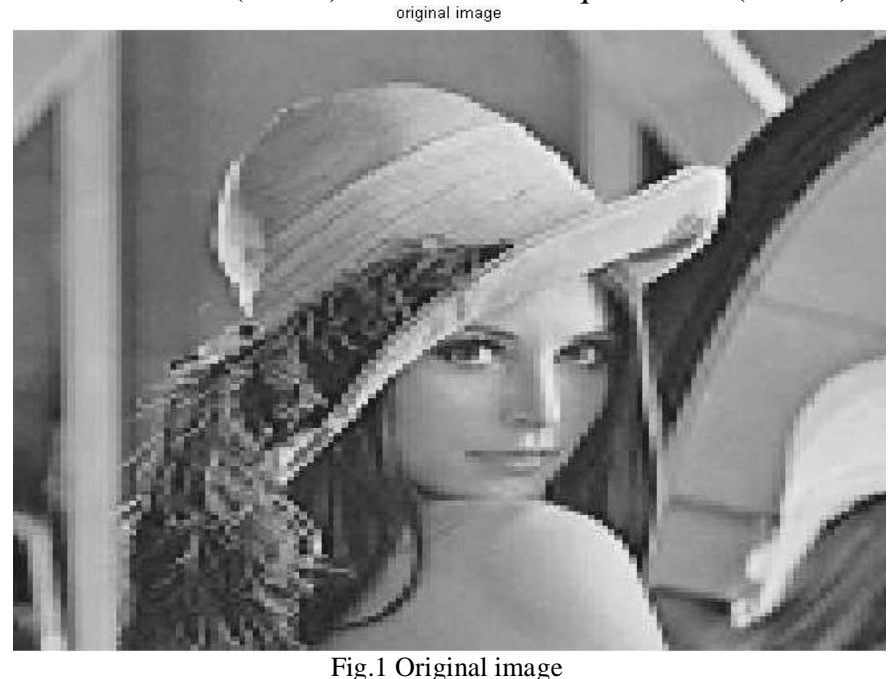

$$
\begin{aligned}
& \text { Fig.1 Original image } \\
& \text { noisy image with gaussian noise }
\end{aligned}
$$

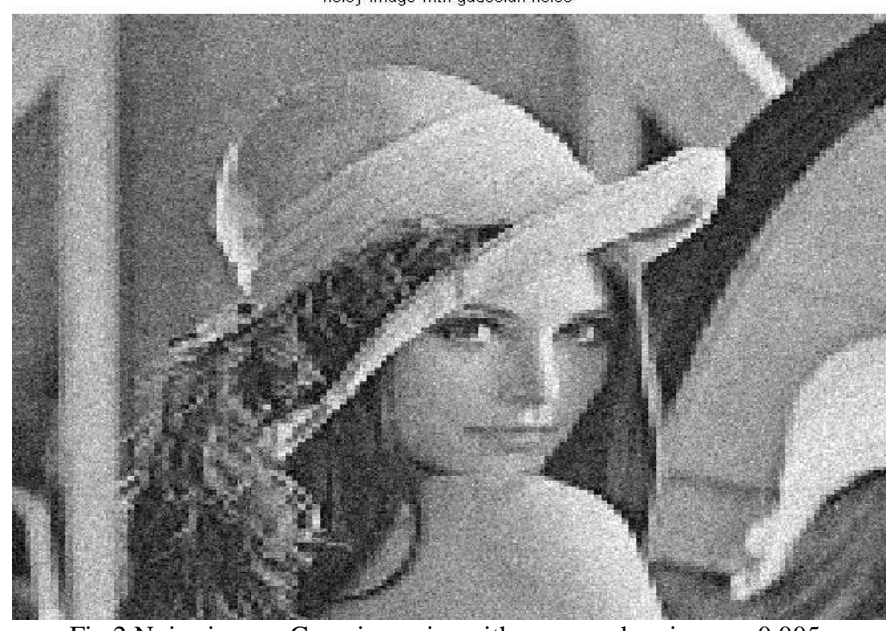

Fig.2 Noisy image: Gaussian noise with mean and variance $=0.005$

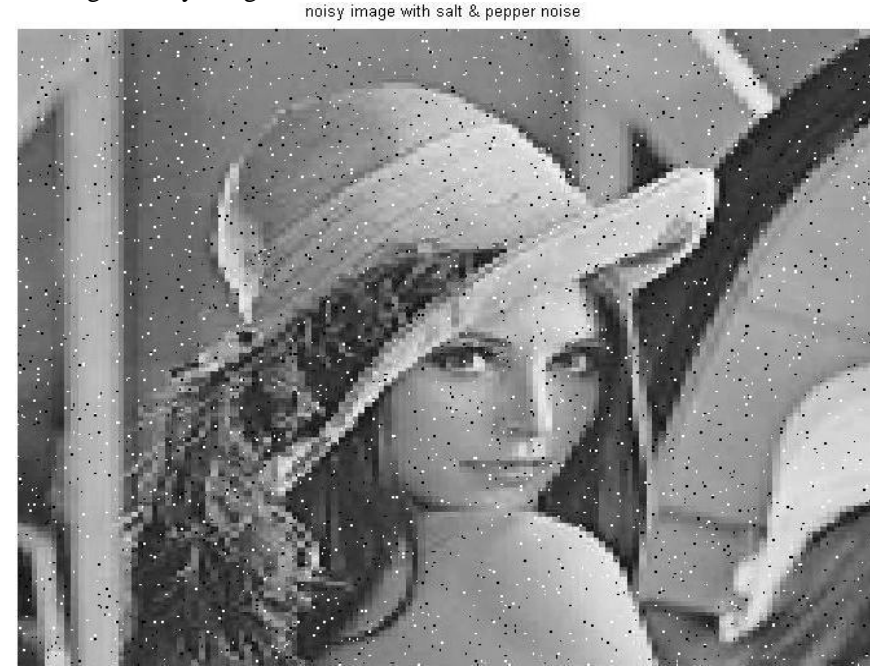

Fig. 3 Noisy image: Salt and pepper noise with noise density $=0.02$ 


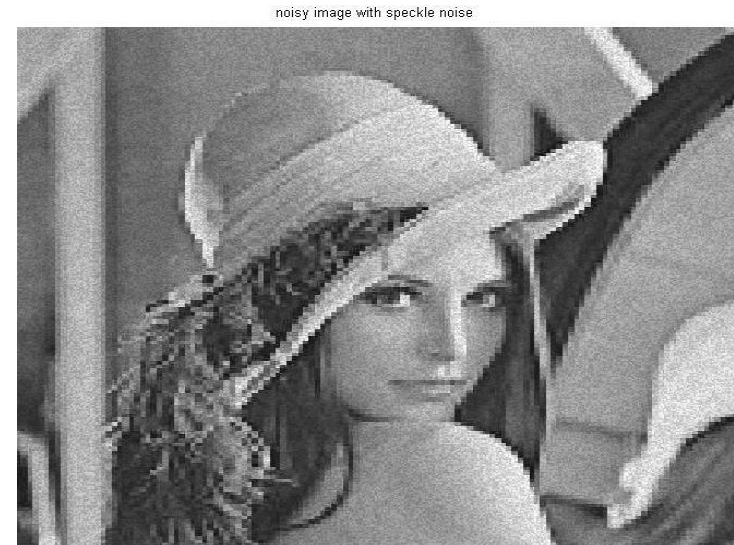

Fig.4 Noisy image: Speckle noise with variance $=0.005$

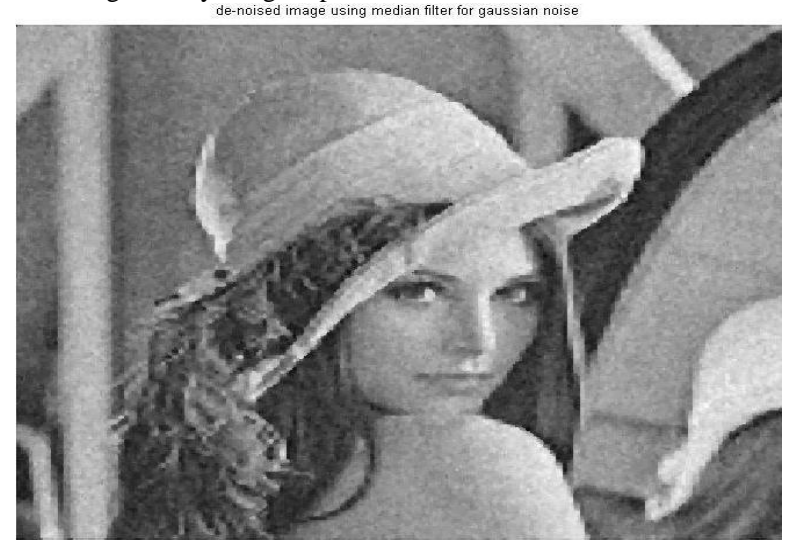

Fig. 5 De-noised image by Median filter for Gaussian noise de-noised image using gaussian filter for gaussian noise

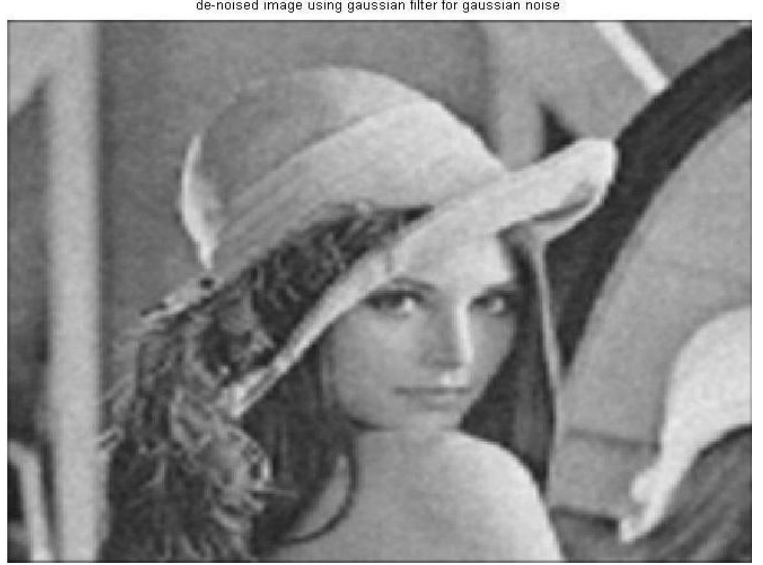

Fig.6 De-noised image by Gaussian filter for Gaussian noise

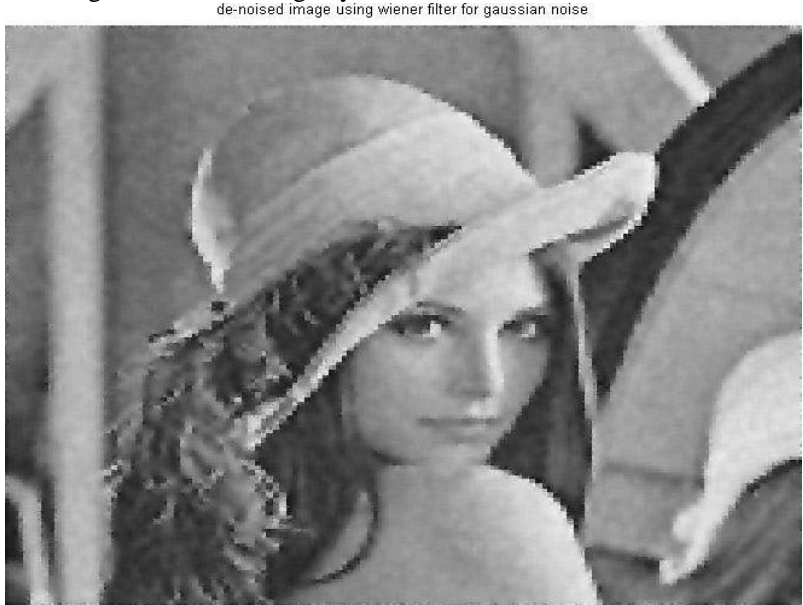

Fig.7 De-noised image by Wiener filter for Gaussian noise 


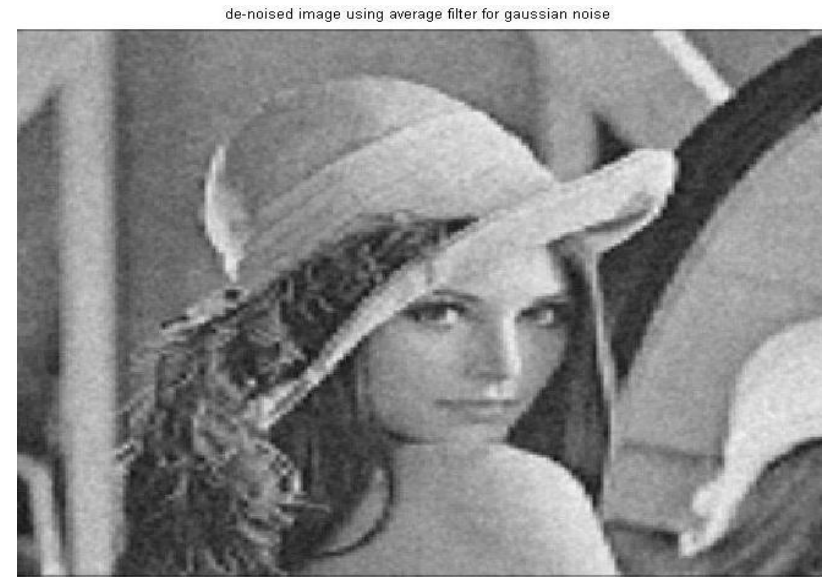

Fig.8De-noised image by Average filter for Gaussian noise

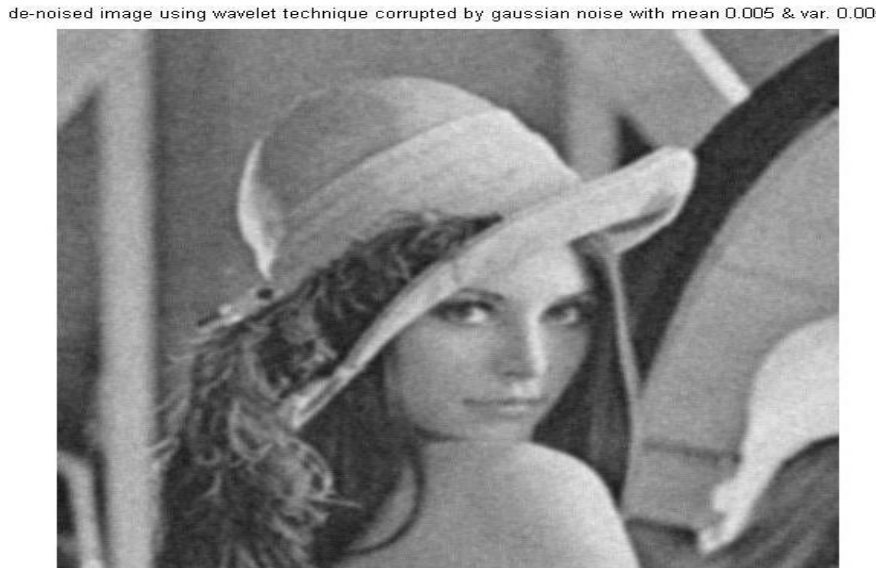

Fig.9 De-noised image using Wavelet Transform for Gaussian noise

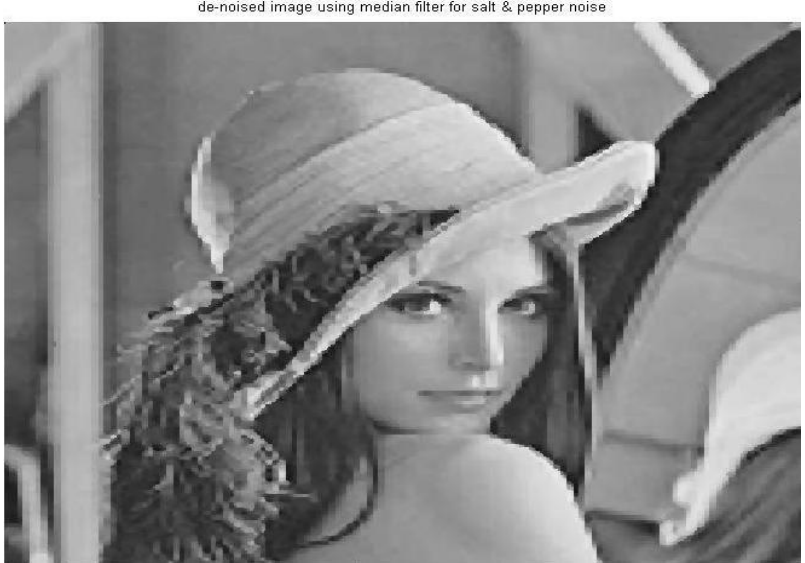

Fig.10De-noised image by Median filter for Salt and pepper noise

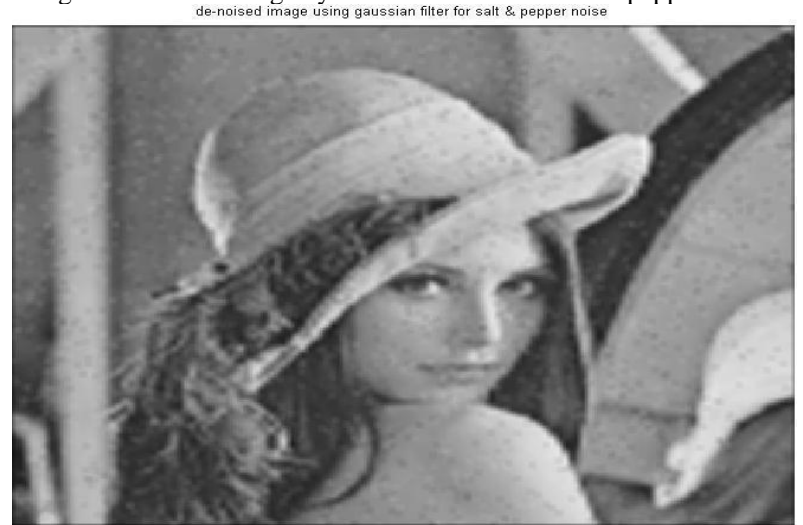

Fig.11De-noised image by Gaussian filter for Salt and pepper noise 


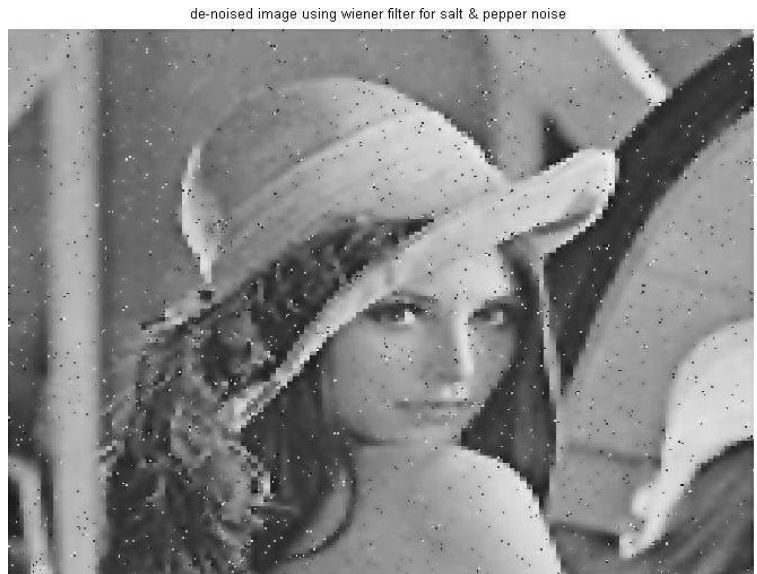

Fig.12De-noised image by Wiener filter for Salt and pepper noise

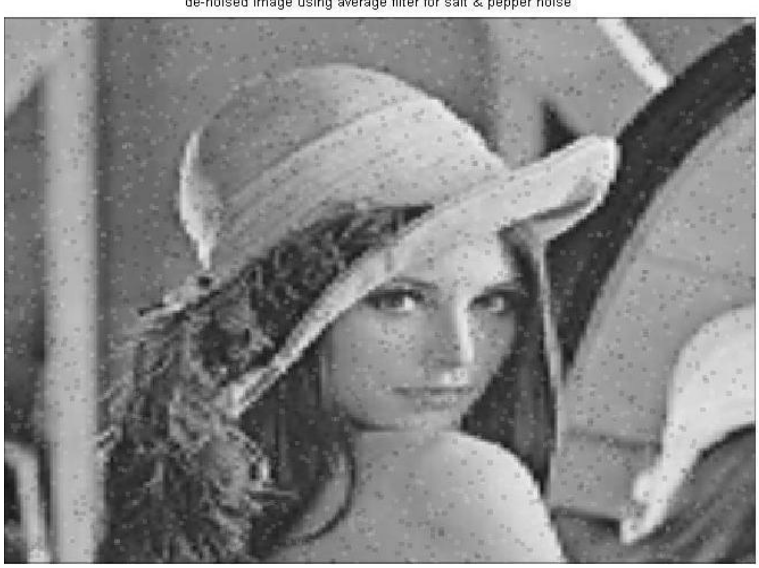

Fig.13De-noised image by Average filter for Salt and pepper noise

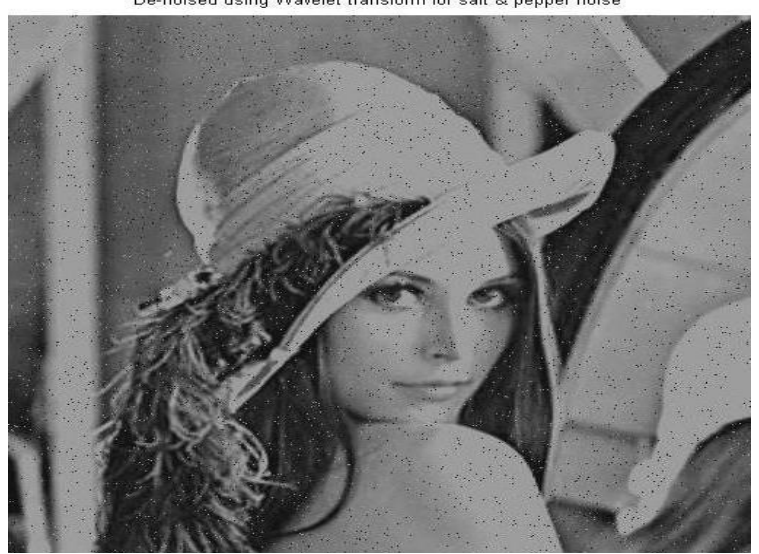

Fig.14 De-noised image using Wavelet Transform for Salt and pepper noise

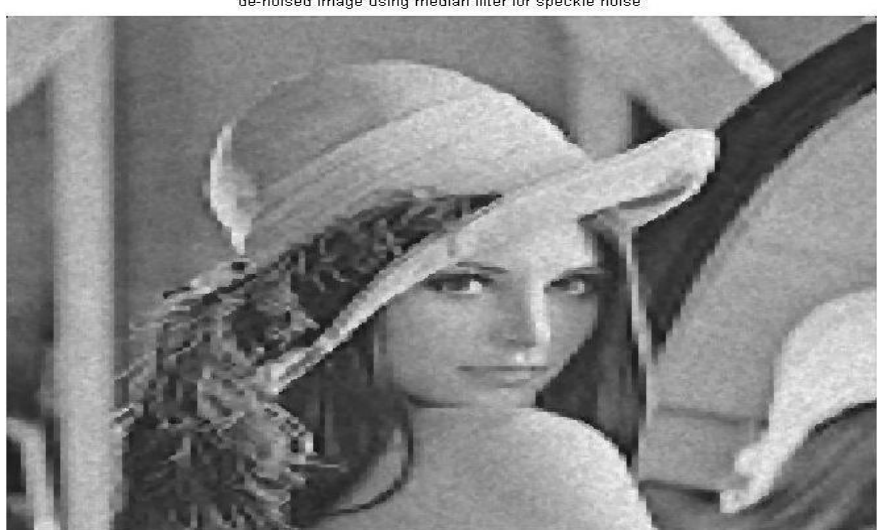

Fig.15De-noised image by Median filter for Speckle noise 


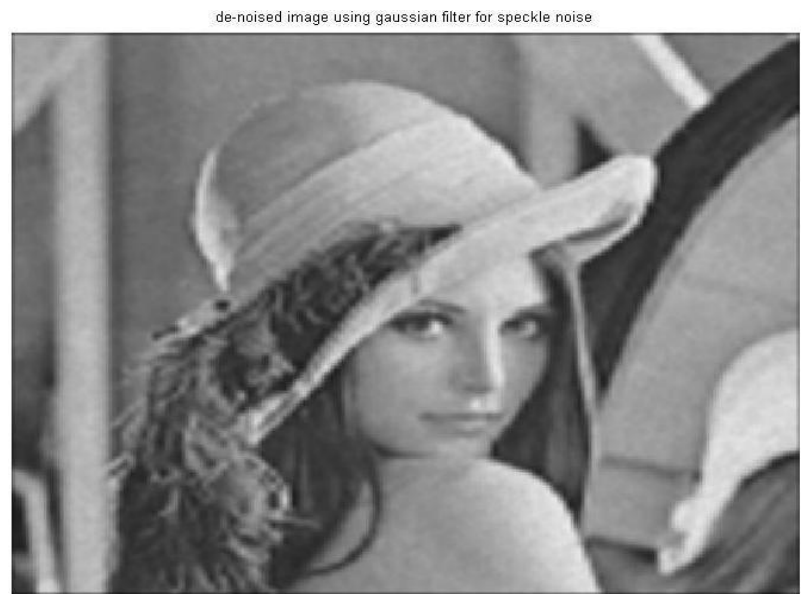

Fig.16De-noised image by Gaussian filter for Speckle noise

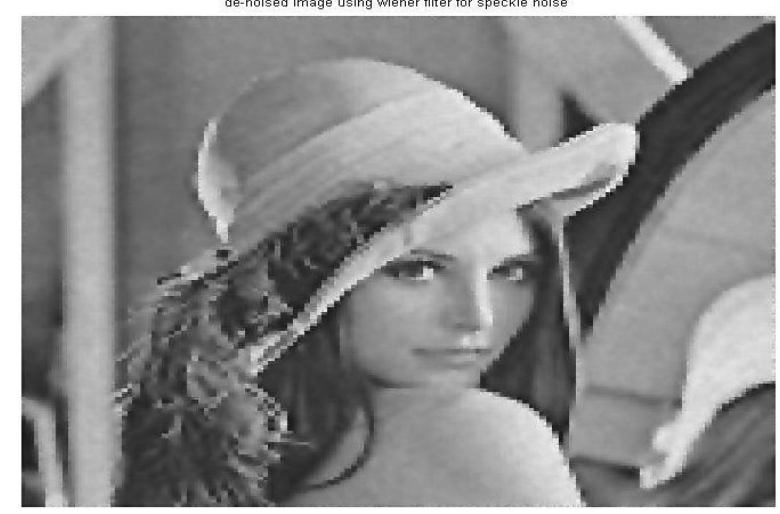

Fig.17De-noised image by Wiener filter for speckle noise

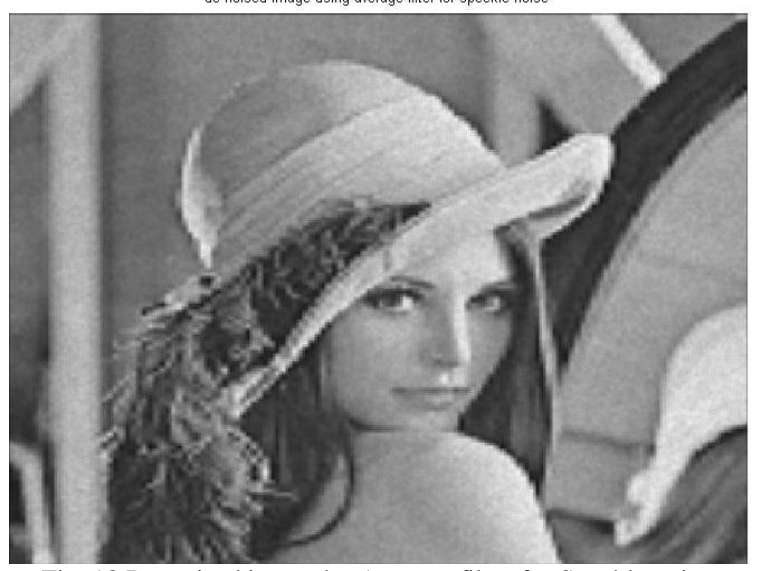

Fig. 18 De-noised image by Average filter for Speckle noise

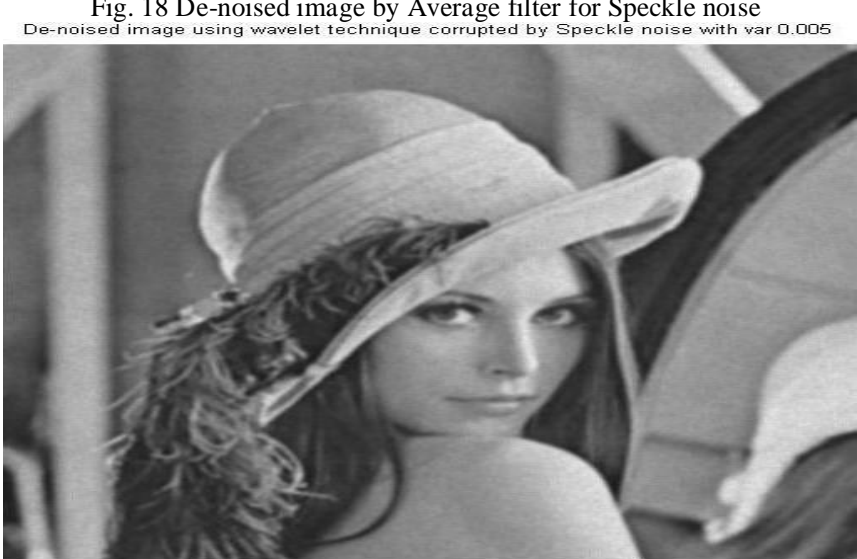

Fig.19 De-noised image using Wavelet Transform for Speckle noise 


\section{RESUlts}

TABLE I

COMPARISON OF PSNR AND RMSE VALUES FOR GAUSSIAN NOISE

\begin{tabular}{|c|c|c|}
\hline & PSNR & RMSE \\
\hline Median filter & 33.0336 & 5.5658 \\
\hline Gaussian filter & 33.2484 & 5.4157 \\
\hline Wiener filter & 34.2525 & 4.8229 \\
\hline Average filter & 33.2805 & 5.4056 \\
\hline Wavelet transform & 38.9509 & 4.0410 \\
\hline
\end{tabular}

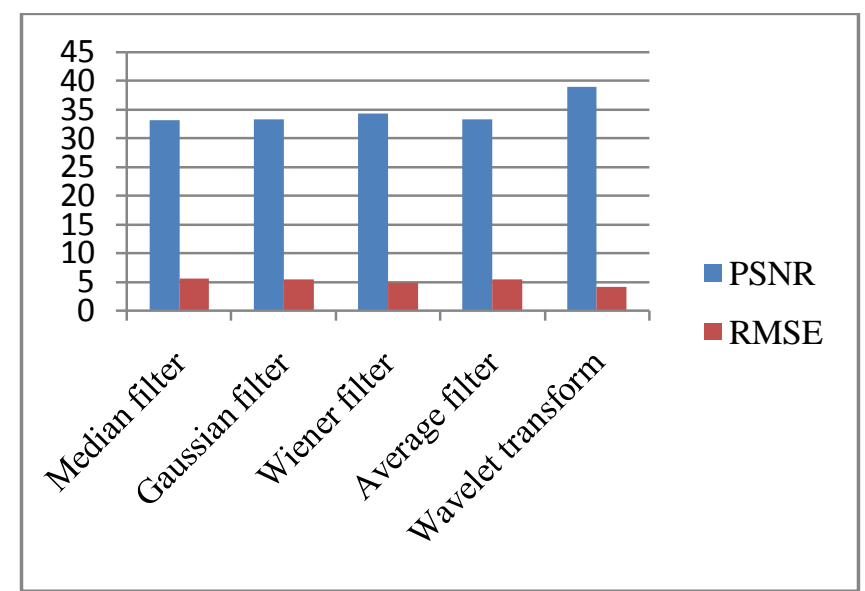

The graph is plotted for PSNR and RMSE values resulting from different filters. This graph shows that Wavelet Transform method is more efficient for removing Gaussian noise than other filters, but performance of Wiener Filter is also good enough for Gaussian noise.

TABLE II

COMPARISON OF PSNR AND RMSE VALUES FOR

\begin{tabular}{|c|c|c|}
\multicolumn{1}{c}{ SALT AND PEPPER NOISE } \\
\hline & PSNR & RMSE \\
\hline Median filter & 37.5965 & 3.2668 \\
\hline Gaussian filter & 32.4363 & 5.9546 \\
\hline Wiener filter & 34.0572 & 5.7428 \\
\hline Average filter & 32.7322 & 4.9548 \\
\hline Wavelet transform & 33.7444 & 4.6306 \\
\hline
\end{tabular}

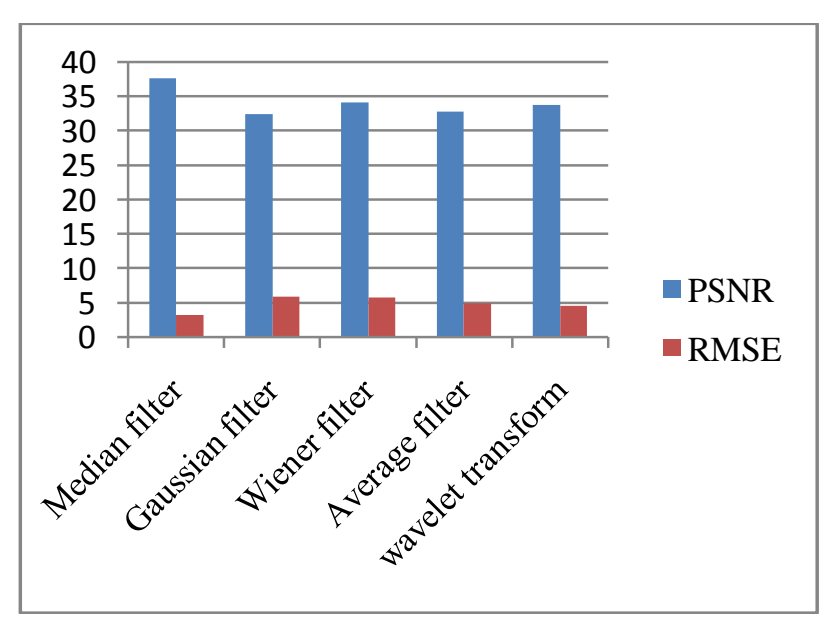

The graph is plotted for PSNR and RMSE values resulting from different filters. The graph shows that median filter is more efficient in removing salt and pepper noise than other filters, but here also Wavelet transform can de-noise image at satisfactory level for salt and pepper noise. 
TABLE III

COMPARISON OF PSNR AND RMSE VALUES FOR SPECKLE NOISE

\begin{tabular}{|c|c|c|}
\hline & PSNR & RMSE \\
\hline Median filter & 33.8149 & 5.0891 \\
\hline Gaussian filter & 33.0550 & 5.5266 \\
\hline Wiener filter & 34.4384 & 4.7228 \\
\hline Average filter & 33.8463 & 5.0450 \\
\hline Wavelet transform & 37.4216 & 3.8463 \\
\hline
\end{tabular}

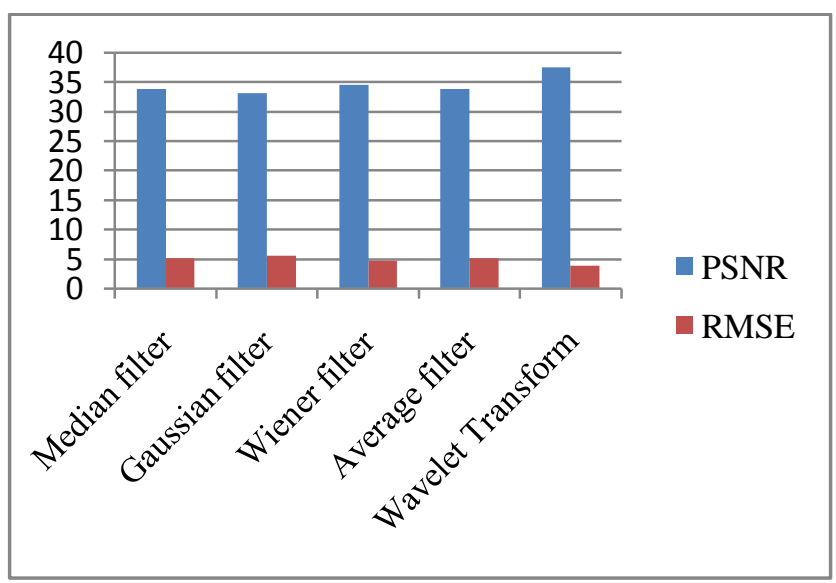

The graph is plotted for PSNR and RMSE values resulting from different filters. This graph shows that Wavelet Transform is more efficient for removing speckle noise than other filters, but also Wiener filter perform good with Speckle noise.

\section{CONCLUSION}

In this paper, filtering method and wavelet transform method is used and evaluated in order to recover an image with noise contaminated effectively. Three types of noise i.e. Gaussian noise, Speckle noise, Salt and pepper noise is added to original image. Wavelet Transform performs better in removing Gaussian noise and Speckle noise than other filters because of its unique signal analysis technique. Median filter performs better with salt and pepper noise. This paper displays the fact that Wavelet Transform can work effectively for any kind of noise by choosing appropriate threshold.In this paper PSNR and RMSE has been used as de-noising parameter. Results have been simulated on MATLAB 2010.

[1] R.C. Gonzalez and R.E. Woods "Digital Image Processing."

\section{References}

[2] K.P. Soman and K.I. Ramachandran, "Insight into Wavelets from Theory to Practice".

[3] M. Sifuzzaman, M.R. Islam, M.Z. Ali, "Application of Wavelet Transform and its Advantages Compared to Fourier Transform", Journal of Physical Sciences, Vol.13,2009.

[4] Suresh Kumar, Papendra Kumar, Manoj Gupta, Ashok Kumar Nagawat, "Performance Comparison of Median and Wiener Filter in Image De-noising”, International Journal of Computer Application, Vol.12 - No.4, November 2010.

[5] Sarita Dangeti, "De-noising Techniques: a Comparison", M.S. Thesis, Louisiana State University, 2003.

[6] S. Grace Chang, Bin Yu, Martin Vetterli, “Adaptive Wavelet Thresholding for De-noising and Compression”, IEEE Transactions on Image Processing, Vol.9, No.9, September 2000.

[7] Javier Portilla, Vasily Strela, Martin J. Wainwright, Eero P. Simoncelly, “ Image De-noising using Scale Mixtures of Gaussians in Wavelet Domain ", IEEE Transactions on Image Processing, Vol.12, No.11, November 2003.

[8] S. Sudha, G. R. Suresh, R. Sukanesh, “Wavelet based Image De-noising using Adaptive Thresholding ”, International Conference on Computational Intelligence and Multimedia Applications, 2007.

[9] J. N. Ellinas, T. Mandadelis, A. Tzortzis, L. Aslanoglou,“ Image De-noising using Wavelets ”, T. E. I. of Piraeus, Department of Electronic Computer Systems.

[10] Shi Zhong, Vladimir Cherkassky, “ Image De-noising using Wavelet Thresholding and Model Selection ”, IEEE, Proceedings 2000 .

[11] Saurabh Kumar Mishra, Kamal Ahmad, Akanksha Trivedi, Mudit Shukla, Harshita Pandey, "Image de-noising using wavelet thresholding method" International Journal of Advanced Scientific and Technical Research, Issue 3 volume 1, January-February 2013. 